\title{
Primary Low Cerebrospinal Fluid Pressure Syndrome Associated with Galactorrhea
}

\author{
Michiya Yamamoto, Tadashi Suehiro, Hideshi Nakata, Tatsuya Nishioka, Hiroyuki Itoh, \\ Toshihiro NaKamura and Kozo Hashimoto
}

\begin{abstract}
A 36-year-old woman with positional headache was found to have primary low cerebrospinal fluid (CSF) pressure syndrome and galactorrhea. The CSF pressure at lumbar puncture was not measurable. Magnetic resonance imaging demonstrated that the ventricle and cerebral sulcus were narrowed and the pituitary stalk was oppressed by the brain. Hyperresponsiveness of prolactin was noted after stimulation with thyrotropin-releasing hormone. These abnormalities disappeared with normalization of CSF pressure with the treatment. Galactorrhea was apparently due to oppression of the pituitary stalk by downward movement of the brain.
\end{abstract}

(Internal Medicine 32: 228-231, 1993)

Key words: low cerebrospinal fluid pressure syndrome, galactorrhea, headache, pituitary stalk

\section{Introduction}

Low cerebrospinal fluid (CSF) pressure syndrome is characterized by a posture-dependent headache and low CSF pressure $(1-3)$. The condition is usually seen after lumbar puncture, cranial surgery, head trauma or meningitis (1). Low CSF pressure rarely develops spontaneously without an obvious precipitation event. This syndrome was first described by Schaltenbrand in 1938 (4), but the etiology has not been clarified.

Here, we report a case of primary low CSF pressure syndrome associated with galactorrhea, and discuss a possible pathogenetic mechanism of this syndrome.

\section{Case Report}

On August 9, 1991 a 36-year-old woman developed severe bilateral headache which was aggravated in the upright position and subsided in the supine position. Subsequently, the posture-dependent headache worsened and was accompanied by nausea induced with nonsteroidal anti-inflammatory drug. Symptoms became intolerable and, on August 16, the patient visited our clinic and was admitted immediately. She had no history of trauma or physical illness, and her menstrual periods had been regular.

There was no mental disturbance. Blood pressure was
$90 / 50 \mathrm{mmHg}$ in the supine position and $90 / 58 \mathrm{mmHg}$ in the sitting position. Discharge of a milk-like substance from both breasts was observed. Neurological examination did not reveal neck stiffness, cranial nerve disturbance or any motor or sensory disturbances.

The results of routine laboratory examination including urinalysis, erythrocyte sedimentation rate, complete blood count, blood chemistry and blood serology were within normal limits. Two days after admission a lumbar puncture was done. CSF pressure was not measurable and there was no spontaneous drop in CSF in the lateral decubitus position. An aspirated sample of CSF was clear, and analysis showed a count of 6 cells/ $\mu \mathrm{l}$ ( 2 granulocytes and 4 lymphocytes), a protein level of $96 \mathrm{mg} / \mathrm{dl}$, glucose of $54 \mathrm{mg} / \mathrm{dl}$ and chloride of $123 \mathrm{mEq} / \mathrm{l}$. Computerized tomography $(\mathrm{CT})$ of the brain demonstrated slit ventricles and narrowing of the cerebral sulcus, Sylvian fissures and basal cisterns. Lumbar isotope cisternography followed for 30 hours showed no evidence of abnormal leakage of dye from the subarachnoid space, though isotope clearance was not performed. On the basis of these findings, a diagnosis of primary low CSF pressure syndrome was made.

Magnetic resonance imaging (MRI) of the brain enhanced by gadolinium demonstrated that the pituitary gland was enlarged and the pituitary stalk was oppressed by the brain (Fig. 1A,C). Baseline plasma adrenocortico-

From the Second Department of Internal Medicine, Kochi Medical School, Nankoku, Kochi

Received for publication September 11, 1992; Accepted for publication January 12, 1993

Reprint requests should be addressed to Dr. Michiya Yamamoto, the Second Department of Internal Medicine, Kohasu, Okoh-cho, Nankoku, Kochi 783, Japan 
tropic hormone $(\mathrm{ACTH})$, cortisol and urinary 17-OHCS were $62 \mathrm{pg} / \mathrm{ml}, 13 \mu \mathrm{g} / \mathrm{dl}$ and $2.2 \mathrm{mg} /$ day, respectively. Serum thyroxine at $118 \mathrm{ng} / \mathrm{dl}$, triiodothyronine at $7.6 \mu \mathrm{g} /$ $\mathrm{dl}$, and thyroid stimulating hormone (TSH) at $1.05 \mu \mathrm{IU} /$ $\mathrm{ml}$ were within normal limits. Basal serum prolactin level of $17.6 \mathrm{ng} / \mathrm{ml}$ was also normal. Delayed responses of growth hormone $(\mathrm{GH})$ and $\mathrm{ACTH}$ were shown in stimulation tests with GH-releasing factor (GRF) and corticotropin-releasing hormone $(\mathrm{CRH})$, respectively (Fig. 2A,B). Luteinizing hormone (LH) and follicule stimulating hormone (FSH) showed low responses to LH-releasing hormone (LH-RH) (Fig. 2C). TSH showed a normal response to thyrotropin-releasing hormone (TRH), but prolactin was hyperresponsive (the maximum level was $436 \mathrm{ng} / \mathrm{ml}$ ) (Fig. 2D).

With bed rest and intravenous transfusions of isotonic solution, the patient's severe headache decreased gradually. Three weeks after the onset, she was able to walk and galactorrhea ceased. However, seven weeks after the onset, the patient still reported a heavy sensation in her head, and CSF pressure at repeat lumbar puncture was still $0 \mathrm{mmHg}$. The patient was discharged on September 20 and was given diphenidol at the outpatient clinic. Nine weeks after the onset, her headache disappeared completely, and a third lumbar puncture showed a normal CSF pressure of $170 \mathrm{mmHg}$ and normal protein level of $67 \mathrm{mg} / \mathrm{dl}$. At this time, pituitary hormone stimulation tests were performed again. The delayed responses of GH and ACTH in GRF and CRH stimulation tests were improved, and the low response of LH in a LH-RH test was normalized. The hyperresponsiveness of prolactin to TRH stimulation was improved. A follow-up MRI showed no enlargement of the pituitary and no oppression of the pituitary stalk, with normalized tight sulcus and ventricle findings (Fig. 1B,D).

\section{Discussion}

This patient had a severe positional headache and unmeasurable CSF pressure without an obvious cause,

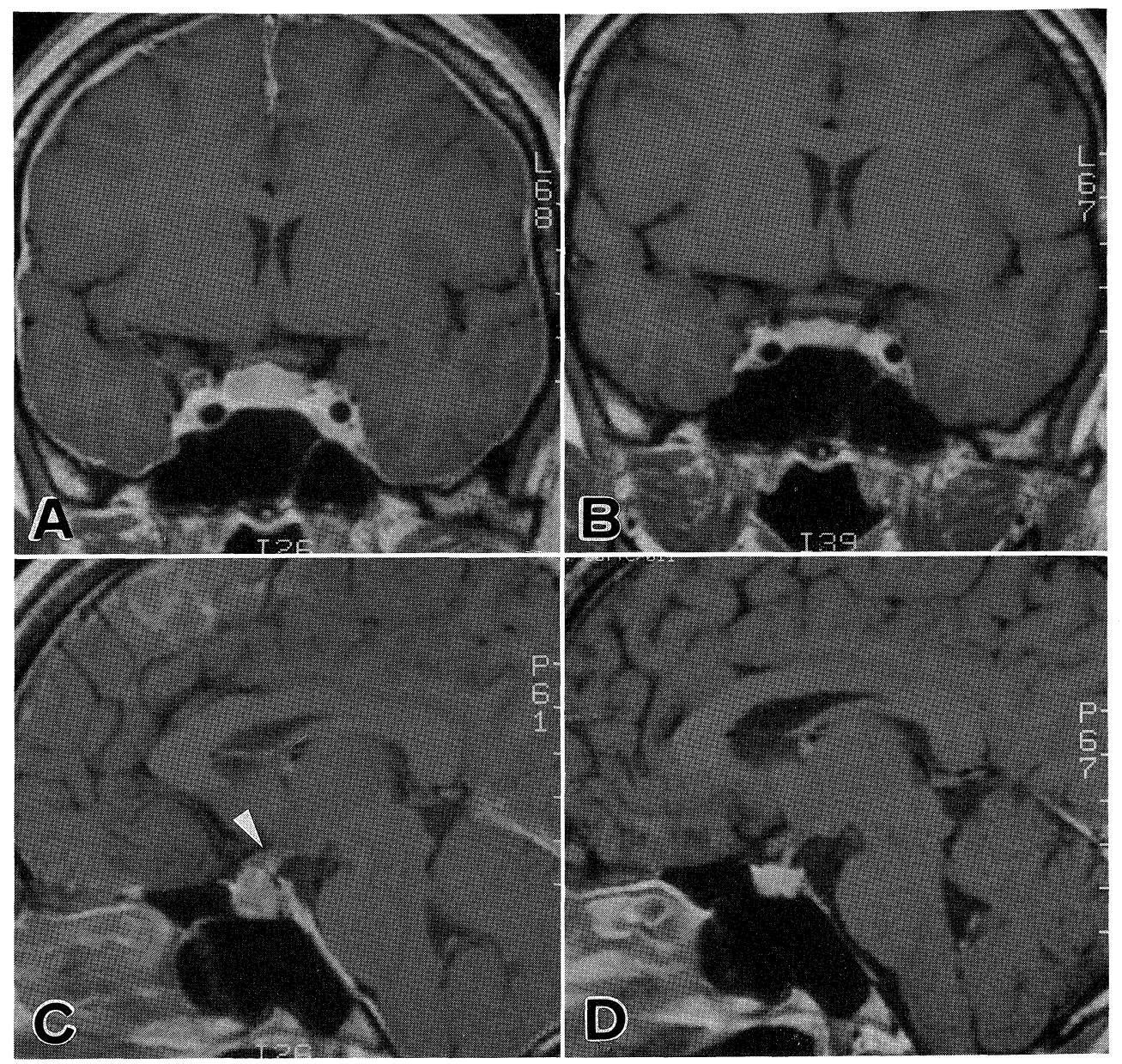

Fig. 1. MRI at two weeks after onset showns tight sulcus, narrow basal cisterns (A), enlarged pituitary and oppression of the stalk (white arrow) (C). MRI at 12 weeks after onset showed cisterns of normal size (B), and normal pituitary and stalk (D). 

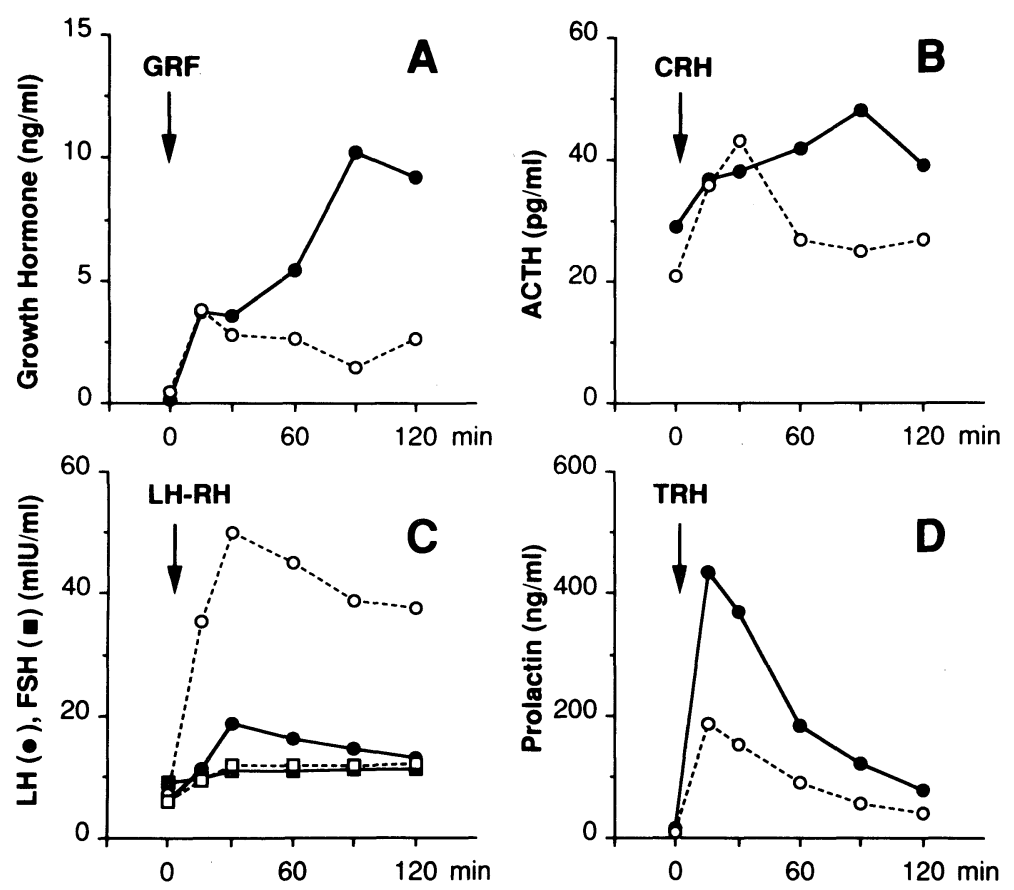

Fig. 2. The responses of GH, ACTH, LH, FSH and prolactin to GRF, CRH, LH-RH and TRH stimulation in this patient. Solid lines: two weeks after onset; broken lines: nine weeks after onset. Delayed responses of GH and ACTH occurred in GRF and $\mathrm{CRH}$ stimulation tests, respectively, during the low-CSF pressure stage (A, B). Low responses of $\mathrm{LH}$ and FSH (C) and hyperresponse of prolactin were also noted. These abnormal responses disappeared after the normalization of CSF pressure.

such as head trauma, cranial surgery, dehydration or cerebral arteriosclerosis $(1,5)$. Therefore, a diagnosis of primary low CSF pressure syndrome was made. This syndrome is rare, but over 70 cases have been reported in the literature (6). However, none of the cases reported have involved galactorrhea, and no detailed examinations of hypothalamic-pituitary hormones have been described in patients with this syndrome.

The characteristic headache accompanying low CSF pressure may be induced by the downward movement of the brain and subsequent traction of pain-sensitive intracranial vessels and dural attachments (1). The $\mathrm{CT}$ and MRI findings of tight Sylvian fissure, narrow basal cisterns $(7,8)$ and oppression of the pituitary stalk suggested that the brain in the present case had moved slightly downwards.

The patient had taken no drugs which might induce galactorrhea, such as estrogens or metoclopramide. The results of hormone stimulation $\mathrm{ACTH}$, suggesting a disorder of the pituitary stalk. Therefore, these data suggest that the hyperprolactinemia in this case was caused by the decrease in prolactin inhibiting factor which induced with oppression of pituitary stalk due to a low CSF pressure.

Although the etiology of primary low CSF pressure syndrome is unknown, it has been assumed that a spontaneous decrease in or cessation of spinal fluid production might be caused by a reversible disorder of vasomotor function of the choroid plexus (9), or by a disorder of the hypothalamus which controls CSF formation (1). It has also been suspected that CSF may leak from tears in the nerve root sleeves, which are not recognized by the patient $(1,10,11)$. Recently, a few studies with lumbar isotope cisternography suggested that a low CSF pressure may be caused by rapid absorption or unusual CSF leakage $(12-14)$. Cisternography in the present case did not show definite leadkage of CSF from the arachnoid plexus and spine, and the patient's CSF pressure normalized after administration of diphenidol which is known to increase CSF production. These results suggested that a decrease in CSF production occurred in this patient. Once the CSF pressure had decreased, the brain may have gravitated downward and oppressed the hypothalamic-pituitary stalk axis, which might have resulted in further reduction in CSF production. This cycle may be the mechanism which sustains low CSF pressure in this syndrome.

In most reported cases of the syndrome, recovery was complete within 2 to 16 weeks (2). However, approximately $10 \%$ of the patients with primary low CSF pres- 


\section{Low CSF Pressure Syndrome}

sure syndrome experienced associated subdural hematoma $(6,15,16)$, presumably because the reduced volume of CSF was compensated by an abnormal engorgement of the vascular system which made the vessels easily damaged. Fortunately, the present case did not involve hematoma. However, the low CSF pressure continued for more than 7 weeks in spite of bed rest and transfusions. Two weeks after administration of diphenidol (17), the patient's CSF pressure normalized. Thus, it may be beneficial to administer such an agent in the early stage of low CSF pressure syndrome.

In conclusion, the findings in this case suggest that the area around the hypothalamic-pituitary axis was damaged by oppression due to downward movement of the brain, resulting in the development of low CSF pressure syndrome and hyperprolactinemia.

\section{References}

1) Bell WE, Joynt RJ, Sahs AL. Low spinal fluid pressure syndromes. Neurology 10: 512, 1960.

2) Diamond S, Baltes BJ. Headache associated with low spinal fluid pressure syndrome (primary intracranial hypotension). Illinois Med J 144: 560, 1973.

3) Baker CC. Headache due to spontaneous low spinal fluid pressure. Minnesota Med 66: 325, 1983.

4) Schaltenbrand G. Neuere anschaugen zur pathophysiologie der liguorzirkulation. Zentralbl Neurochir 3: 290, 1938 (in German).

5) Shenkin HA, Finneson BE. Clinical significance of low cerebral spinal fluid pressure. Neurology 8: 157, 1958.

6) Kishikawa K, Nakae Y, Fujiwara S, Namiki A. Headache due to spontaneous low cerebrospinal fluid pressure. The Pain Clinic 3:
97, 1990.

7) Murros K, Foglholm. Spontaneous intracranial hypotension with slit ventricles. J Neurol Neurosurg Psychiatry 46: 1149, 1983.

8) Hoshino M, Okayama K, Kubo H, Watanabe H, Endou R. CT study in primary low spinal fluid pressure syndrome. Clin Neurol 31: 142, 1991 (in Japanese with English abstract).

9) Schaltenbrand G. Normal and pathological physiology of the cerebrospinal fluid circulation. Lancet 1: 805, 1953.

10) Laseter GM. Primary intracranial hypotension: The low spinal fluid pressure syndrome. Headache 10: 63, 1970.

11) Lipman IJ. Primary intracranial hypotension. The syndrome of spontaneous low cerebrospinal fluid pressure with traction headache. Dis Nerv Syst 38: 212, 1977.

12) Labadie EL, Antwerp JV, Bamford CR. Abnormal lumbar isotope cisternography in an unusual case of spontaneous hypoliquorrheic headache. Neurology 26: 135, 1976.

13) Petersen RC, et al. Successful treatment of spontaneous low cerebrospinal fluid pressure headache. Ann Neurol 22: 148, 1987 (abstract).

14) Hoshino M, Okayama K, Kubo H, Tei H, Watanabe H. A case of primary low spinal fluid pressure syndrome complicated by bilateral subdural hematoma - Study of the cerebrospinal fluid circulation. Clin Neurol 30: 174, 1990 (in Japanese with English abstract).

15) Sipe JC, Zyroff J, Waltz TA. Primary intracranial hypotension and bilateral isodense subdural hematomas. Neurology 31: 334, 1981.

16) Ohara K, Seki $Y$, Maeda T, Aiba T. Primary intracranial hypotension associated with chronic subdural hematoma - Report of two cases. Neurosurgery 12: 1203, 1984 (in Japanese with English abstract).

17) Tsukamoto S, Takemura K, Hori H, Horiike N, Kyoi K, Utsumi S. Clinical studies of diphenidol (Cephadol) in patients with low cerebrospinal fluid pressure. Shinyaku to Rinsyo 29: 1729, 1980 (in Japanese). 